\title{
El subjuntivo en español e italiano: una descripción contrastiva*
}

FRANKLIN YESSID ARIAS BEDOYA**

Recepción: 22 de abril de 2021

Aprobación: 15 de julio de 2021

Forma de citar este artículo: Arias, B. (2021). El subjuntivo en español e italiano: una descripción contrastiva. Cuadernos de Lingüística Hispánica, (37), e12798.

* Este artículo de investigación surge de la tesis "Análisis contrastivo del subjuntivo en español e italiano", dirigida por las doctoras Chiara Landi y Franca Orletti.

** Licenciado en Educación Básica con Énfasis en Humanidades, Lengua Castellana de la Universidad de Antioquia, Colombia. Laureato in lingua e cultura italiana per stranieri en la Università degli studi di Pisa,Italia. Magíster en Lingüística de la Università degli Studi di Roma, La Sapienza, Italia. f.ariasbedoya@studenti.unipi.it. :2: https://orcid.org/0000-0001-5415-0897 


\section{Resumen}

En el panorama lingüístico hispanófono e italófono, el subjuntivo ha sido definido principalmente desde una perspectiva estructural con base en la noción de dependencia sintáctica. A partir del siglo XIX el enfoque semántico cobró relevancia y las nociones de realidad / irrealidad comenzaron a predominar en la definición de modo. Sin embargo, numerosas fuentes bibliográficas como Vásquez (2013), Stewart (2002) y Haverkate (2002) han revelado que el uso del subjuntivo no solo se limita a la aparición de un modalizador de posibilidad, hecho imaginario o potencial, sino que la intención del hablante y la presencia de inductores sin ningún valor de irrealidad pueden introducir formas de subjuntivo inexplicables desde una perspectiva puramente semántica o netamente sintáctica. El objetivo del presente artículo es identificar las principales distribuciones del subjuntivo en español e italiano. El análisis contrastivo, exploratorio, descriptivo y documental se limita a proposiciones completivas epistémicas y deónticas, oraciones relativas y subordinadas adverbiales, dadas las limitaciones de espacio.

Palabras clave: subjuntivo, italiano, español, lingüística contrastiva.

\section{The subjunctive in spanish and italian: a contrastive description}

\section{Abstract}

In the Spanish-speaking and Italian-speaking linguistic panorama, the subjunctive has been defined mainly from a structural perspective based on the notion of syntactic dependency. From the 19th century became relevant the semantic approach, and the notions of reality / unreality began to predominate in the definition of mode. However, many bibliographic sources such as Vásquez (2013), Stewart (2002) and Haverkate (2002), have revealed that the use of the subjunctive is not only limited to the appearance of a modalizer of possibility, imaginary, or potential fact, but that the intention of the speaker and the presence of inducers without any unreality value can introduce inexplicable forms of the subjunctive from a perspective purely semantic or clearly syntactic.

The objective of this paper is to identify the main distributions of the subjunctive in Spanish and Italian. Contrastive, exploratory, descriptive, and documentary analysis is limited to epistemic and deontic completive propositions, relative sentences and adverbial subordinate clauses, given space limitations.

Keywords: subjunctive, Italian, Spanish, contrastive linguistics. 


\section{Le subjonctif en espagnol et italien: une description contrastive}

\section{Résumé}

Dans le paysage linguistique hispanophone et italophone, le subjonctif a été défini principalement dans une perspective structurelle basée sur la notion de dépendance syntaxique. À partir du XIXe siècle, l'approche sémantique a gagné en pertinence et les notions de réalité/irréalité ont commencé à prédominer dans la définition de mode. Cependant, de nombreuses sources bibliographiques telles que Vásquez (2013), Stewart (2002) et Haverkate (2002) ont révélé que l'utilisation du subjonctif ne se limite pas seulement à l'occurrence d'un modificateur de possibilité, de fait imaginaire ou de potentiel, mais que l'intention du locuteur et la présence d'inducteurs sans valeur d'irréalité peuvent introduire des formes du subjonctif inexplicables d'un point de vue purement sémantique ou purement syntaxique. L'objectif du présent article est d'identifier les principales distributions du subjonctif en espagnol et en italien. L'analyse contrastive, exploratoire, descriptive et documentaire se limite aux propositions complétives épistémiques et déontiques, aux clauses relatives et aux clauses subordonnées adverbiales, compte tenu des limites d'espace.

Mots-clés: subjonctif, italien, espagnol, linguistique contrastive.

\section{O subjuntivo em espanhol e italiano: uma descrição contrastiva}

\section{Resumo}

No panorama linguístico espanhol e italiano, o subjuntivo foi definido principalmente a partir de uma perspectiva estrutural a partir da noção de dependência sintática. A partir do século XIX, a abordagem semântica ganhou relevância e as noções de realidade / irrealidade passaram a predominar na definição de modo. No entanto, inúmeras fontes bibliográficas como Vásquez (2013), Stewart (2002) e Haver Kate (2002) revelaram que o uso do subjuntivo não se limita apenas ao aparecimento de um modalizador de possibilidade, fato imaginário ou potencial, mas que 0 a intenção do falante e a presença de indutores sem qualquer valor de irrealidade podem introduzir formas subjuntivas inexplicáveis de uma perspectiva puramente semântica ou puramente sintática. 0 objetivo deste artigo é identificar as principais distribuições do subjuntivo em espanhol e italiano. A análise contrastiva, exploratória, descritiva e documental limita-se a proposições completivas epistêmicas e deônticas, orações adverbiais relativas e subordinadas, dadas as limitações de espaço.

Palavras-chave: subjuntivo, italiano, espanhol, linguística contrastiva. 


\section{Introducción}

La variedad lingüística privilegiada durante los procesos de aprendizaje de una lengua extranjera es generalmente la estándar. Berruto (2010) la define como la lengua media, sin rasgos sociolingüísticos y que toma en consideración criterios de codificación normativa, generalización, elaboración e invariabilidad (uniformidad). Esta observación se aplica al español, un sistema que delimita una variedad de código neutro resguardado institucionalmente por la Real Academia Española. La norma lingüística italiana contemporánea, en cambio, se describe con el neoestándar (Berruto, 1987) o italiano de uso medio (Sabatini, 1985), un modelo lingüístico más amplio, nacido durante el proceso de masificación de la mitad del siglo XX y en el que se incluyen elementos previamente considerados subestándar o marcados sociolingüísticamente.

A pesar de la planificación y normalización lingüísticas, el habla cotidiana como sistema dinámico manifiesta múltiples transgresiones con respecto a la norma prescriptiva. Estas variaciones plantean distintas dificultades para los estudiantes extranjeros que aprenden la variedad estándar (español) o neoestándar (italiano). Con este texto se propone realizar un análisis contrastivo entre el subjuntivo italiano y el subjuntivo español desde una perspectiva descriptiva y no meramente normativa. El objetivo del estudio es, por tanto, determinar las principales similitudes y diferencias entre los dos sistemas lingüísticos, teniendo en cuenta los aspectos semántico, sintáctico y pragmático.

\section{Metodología}

El proyecto contempla un diseño no experimental, de tipo documental y de alcance descriptivo y contrastivo. Metodológicamente, el estudio se limita a proposiciones completivas epistémicas y deónticas, cláusulas de relativo y subordinadas adverbiales; se evitan aquellas construcciones en las que el imperativo introduce el subjuntivo y el subjuntivo independiente. El trabajo se divide en tres partes: en el primer capítulo se realizará una caracterización del subjuntivo desde un punto de vista histórico y gramatical. En la segunda sección se examinarán los tipos de proposiciones previamente enunciados, utilizando distintos ejemplos que fueron cotejados por cinco hablantes nativos de ambas lenguas para garantizar la gramaticalidad. Finalmente, se expondrán algunas conclusiones y se indicará un camino por seguir para futuros estudios.

\section{Marco teórico}

\section{Caracterización y definición del subjuntivo}

\section{El problema del modo en la tradición gramatical española}


La definición del subjuntivo siempre ha representado un problema de estudio para los gramáticos españoles, quienes han debatido largamente sobre las delimitaciones de los modos verbales y sus principales características. En efecto, las gramáticas españolas presentan conceptos y definiciones que no han logrado llenar completamente las lagunas conceptuales existentes.

En el siglo XIX, por ejemplo, Salvá (1930) desarrolló una explicación semántico-sintáctica del modo, que concibió como "La manera con que al hablar consideramos la significación del verbo" (p. 51). Según el autor, el indicativo expresaba la idea del verbo sin depender de otro vocablo, mientras que el subjuntivo debía estar unido a un verbo principal "explícito, o sobreentendido que lo determinaba y con el cual se enlazaba por medio de alguna partícula conjuntiva" (Salvá, p. 52). Este enfoque, proveniente de la tradición latina, también se puede verificar en la Gramática Castellana de Nebrija (1492), en la que el subjuntivo se limita a la hipotaxis y en la que la semántica no posee ninguna importancia 0 , a lo sumo, un valor secundario. Sin embargo, esta asociación del indicativo con oraciones independientes y del subjuntivo con oraciones subordinadas es obsoleta.

Andrés Bello (1857), por su parte, entiende como modo "la forma que toma el verbo para indicar operaciones del entendimiento o emociones del ánimo" (p. 111). El gramático distingue entre cuatro paradigmas verbales: el indicativo utilizado en cláusulas dependientes o independientes; el subjuntivo común de proposiciones hipotácticas para representar la duda, la incertidumbre u otras emociones del alma; el subjuntivo bipotético utilizado en oraciones dependientes y que indican una condición o hipótesis; y el subjuntivo optativo usado en cláusulas independientes y que designan un deseo 0 una orden (actual imperativo).

Lenz (1920) expone una definición semántica del modo en la que identifica tres tipologías en función de su contenido lógico: el indicativo o modo de la realidad y los hechos (juicio asertivo); el imperativo, que alude a proposiciones de deseo o lógicamente necesarias (juicio apodíctico); y finalmente el subjuntivo, en el que se manifiesta una posibilidad, hecho imaginario o duda (juicio problemático). Estas dos últimas formas "enuncian los hechos como existentes solo en nuestra imaginación” (Lenz, 1920, p. 452).

La concepción del subjuntivo como un modus irrealis de la subjetividad ha sido explotada por numerosos autores en el siglo XX. Emilio Ridruejo (1999) nos ofrece un resumen sobre el tema: "el subjuntivo se ha descrito como el modo de la no-realidad (Alarcos Llorach, 1994), de la incertidumbre (Badía Margarit, 1953), de la subjetividad (Hernández Alonso, 1984), de la futuridad indefinida (Beardsley, 1921), de lo prospectivo (Charaudeau, 1971), etc., frente al indicativo, modo de la realidad, de la objetividad, de lo seguro o de lo actual" (p. 3218). Gili (1998) concuerda con esta línea de pensamiento, pero contrario 
a Lenz, sostiene que los modos dependen de la actitud (punto de vista) del hablante hacia su enunciado y no del contenido lógico. Gili plantea que los verbos pueden designar acciones objetivas, realmente realizadas (indicativas) o conceptos que son solo actos de la mente y que no tienen existencia fuera del pensamiento. El erudito divide este modo verbal en potencial (dudar, ignorar, temer, sentir que, ser posible) y optativo (ser necesario, querer).

En conclusión, son dos los principales problemas que se pueden verificar en la historia del concepto de modo en la tradición española. El primero es la circunscripción del término a la noción de actitud, que reduce este fenómeno a la subjetividad del hablante:

el modo se usa para establecer ciertos actos verbales, (...) para determinar el grado de compromiso del hablante con lo que afirma, (...) para marcar obligadamente el régimen que corresponde a las oraciones que complementan a determinados predicados, para indicar la (in) especificidad del grupo nominal indefinido. (Real Academia Española, 2010, pp. 473-474)

El segundo problema es la determinación del subjuntivo a partir de la dicotomía realidad / irrealidad, certeza / incertidumbre, compromiso del hablante con la veracidad del enunciado / ausencia de afirmación:

así, en ciertos contextos sintácticos que dejan en suspenso la veracidad de la información proposicional se prefiere el indicativo, a diferencia de lo que sería de esperar (1). Por otro lado, se expresan hechos considerados reales (no hipotéticos) en oraciones con verbo en subjuntivo (2). (Real Academia Española, 2010, p. 473)

(1) -Depende de si viene 0 no

(1)-Dipende dal fatto che viene* / venga o meno.

-Credo che lei viene / venga domani.

(2) -No me gusta que use esa ropa

(2)-Non mi piace che indossi quei vestiti

\section{El subjuntivo en la tradición gramatical italiana}

Desde su origen, las gramáticas italianas han definido el subjuntivo basándose en la noción de dependencia sintáctica. Stewart (2002) menciona que salvo algunos intentos de gramáticos como Ruscelli (1581), Buonmattei (1643) y Soave (1771), el subjuntivo dependiente dominó el panorama lingüístico italiano hasta mediados del siglo XIX. Paralelamente a la tradición española, el final de la década marcó un cambio conceptual hacia un enfoque semántico. Fornaciari (1979), por ejemplo, lo presenta como el modo de la posibilidad, una línea similar que sigue Zambaldi (1905) cuando atestigua 
que "il congiuntivo è il modo dell'azione pensata, cioè di quella che si vuole, si desidera, si suppone, si mette in dubbio" (p. 80).

Cronológicamente se pueden individuar otras definiciones en las que el estereotipo del subjuntivo asociado a la incertidumbre y la irrealidad encuentra espacio en las gramáticas italianas modernas. Battaglia y Pernicone (1980) sostienen que "Il congiuntivo è il modo della possibilità o dell'incertezza. Si adopera, per lo più nelle proposizioni dipendenti, quando l'azione o lo stato indicati dal verbo si presentano alla coscienza di chi parla o scrive come possibili, desiderabili, probabili o incerti" (p. 280) ${ }^{1}$. Más recientemente, Wandruszka (2001) delimita entre las funciones del subjuntivo "quella di distinguere se i contenuti espressi sono affermati come fatti o presentati solo come raffigurazioni soggettive, ad esempio, desideri o supposizioni” (p. 415). El autor además introduce un elemento innovativo dentro del panorama lingüístico italiano, el término subjuntivo de la realidad. En el uso fáctico o temático de la modalidad, la verdad de la oración dependiente es presupuesta por el hablante y se explicita con una oración principal comentativa introducida por construcciones verbales como mi dispiace (siento que), sono felice che, y mi rallegra che (me alegra que). El lingüista advierte que, aunque su noción puede ser comparable al concepto de congiuntivo soggettivo (subjuntivo subjetivo), tal término puede inducir al error, ya que la proposición secundaria es real y no contiene nada de subjetivo.

\section{Los tiempos del modo subjuntivo}

El paradigma de los tiempos verbales dentro del subjuntivo exhibe múltiples equivalencias en las lenguas de estudio. A diferencia de las dos formas en desuso del futuro en español, la misma distribución sintáctica se conserva en ambos sistemas lingüísticos.

La selección verbal se realiza según criterios sintácticos y semánticopragmáticos. En el primer caso, y analizado desde una perspectiva estructural, la elección del tiempo se somete a la denominada consecutio temporum, es decir "l'insieme delle condizioni che regolano i rapporti tra il Tempo verbale di una frase principale (o reggente) e il Tempo verbale di una frase ad essa subordinata" (Vanelli, 2001, p. 612). Estas condiciones atienden aspectos como la conjugación del verbo dominante, las restricciones del verbo principal $^{2}$ y la relación temporal expresada en la oración: "it is a well-known fact that with

1 Tales declaraciones se pueden encontrar además en Sorrento (1951), Dardano y Trifone (1988), Fogarasi (1983) y Sensini (1990). Para obtener más información, consulte Stewart (2002).

2 Según Rojo (1976), existen verbos que no permiten el uso de ciertos tiempos en contextos sintácticos particulares. Aunque sean gramaticalmente correctos, su significado restringe la aparición de algunas formas del paradigma. En oraciones como "Me ordenó que le compre, comprara, *bubiese comprado un libro" / "Mi ha ordinato che gli compri, comprasse, *avessi comprato un libro", el acto de ordenar (forzar), implica una acción potencial y excluye una acción temporalmente precedente al momento de enunciación. 
subjunctive temporal agreement is enforced, i.e., the embedded form must appear in the past or in present, depending upon the form of the superordinate verb: present under present and past under past" (Giorgi, 2009, p. 7). El siguiente esquema ejemplifica las correlaciones prototípicas más frecuentes del subjuntivo:

\begin{tabular}{|c|c|}
\hline \multicolumn{2}{|c|}{ Frase principal en presente } \\
\hline Spero che & Espero que \\
\hline \multicolumn{2}{|c|}{ Posterioridad } \\
\hline $\begin{array}{l}\text { Presente } \\
\text { Futuro }\end{array}$ & Presente \\
\hline \multicolumn{2}{|c|}{ Contemporaneidad } \\
\hline Presente & Presente \\
\hline \multicolumn{2}{|c|}{ Anterioridad } \\
\hline Passato o imperfetto & Pretérito perfecto \\
\hline
\end{tabular}

\begin{tabular}{|c|c|}
\hline \multicolumn{2}{|c|}{ Frase principal en pasado } \\
\hline Volevo che & Quería que \\
\hline \multicolumn{2}{|c|}{ Posterioridad } \\
\hline Imperfetto \\
$\begin{array}{c}\text { Condizionale } \\
\text { composto }\end{array}$ & Pretérito imperfecto \\
\hline \multicolumn{2}{|c|}{ Contemporaneidad } \\
\hline Imperfetto & Pretérito imperfecto \\
\hline \multicolumn{2}{|c|}{ Anterioridad } \\
\hline Piuccheperfetto & Pretérito \\
\hline
\end{tabular}

Figura 1. Consecutio temporum

Sin embargo, Gili (1980) y Rojo (1976) manifiestan que la concordantia temporum no es rígida y que puede por lo tanto variar en el lenguaje hablado. Concretamente, Gili afirma que "un verbo de voluntad como mandar, al hallarse en pasado, puede llevar el subordinado en pretérito imperfecto de subjuntivo (le mandaron que estudiase), pero también en presente (le mandaron que estudie)" (Gili, 1980, p. 281). El italiano, en cambio, prefiere la variación modal a la temporal; este es el caso de las oraciones sustantivas con un verbo modalizador en presente, en el que se puede emplear el presente de subjuntivo o el futuro simple dependiendo del nivel de confiabilidad de la oración (Temo che non arrivi in tempo / Temo che non arriverà in tempo).

En segundo lugar, la selección del tiempo responde a normas semántico-pragmáticas. "The dependency thesis is not mere selection by a higher predicate, but claims that the subjunctive is "triggered" by certain semantic properties of the embedding context, pretty much the way polarity items (PIs) are triggered by their licensers" (Giannakidou, 2009, p. 1883). Esto sucede sobre todo en proposiciones hipotácticas regidas por elementos no verbales, es decir, donde no existe relación de subordinación con un verbo. Haverkate (2002) afirma que en subordinados volitivos introducidos por adverbios de duda como quien, ojalá o así, "the present subjunctive indicates the possible fulfillment of the wish, the imperfect subjunctive 
indicates that its fulfillment is improbable or impossible, and the pluperfect subjunctive indicates that the fulfillment is impossible" (p. 33). En otras palabras, el presente del subjuntivo en frases autónomas expresa un deseo que se considera alcanzable (ojalá venga / *magari venga); el imperfecto, en cambio, indica una situación como incierta o improbable (ojalá viniera / magari venisse); y el pluscuamperfecto se refiere a una acción no realizable porque no ocurrió en el pasado (ojalá hubiera venido / magari fosse venuto). El uso del presente en italiano para hacer esta diferenciación es inadmisible, "desde el punto de vista sintáctico, en italiano magari siempre se construye con el imperfecto o pluscuamperfecto de subjuntivo" (Falcinelli, 2015, p. 34).

\section{Análisis y discusión: la estructura modal en las frases subordinadas}

Proposiciones sustantivas

\section{Predicados de duda, negación y (des)conocimiento (subjuntivo epistémico)}

En los predicados epistémicos, el indicativo alude a una actitud positiva del hablante hacia el valor real de la oración en la que aparece. Mientras tanto, el subjuntivo, cargado de cierta neutralidad, "abarca actitudes epistémicas que van desde la inseguridad en el valor veritativo a la sugerencia 0 implicatura de que tal valor es de falsedad" (Bustos, 1986, p. 252).

En primer lugar, las proposiciones sustantivas se construyen generalmente con el subjuntivo cuando el verbo de la oración principal es dubitare / dudar.

(3) Mi hermana duda que eres / seas inteligente

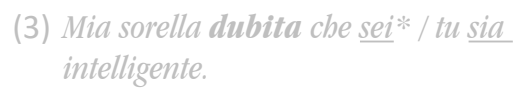

(4) No dudo de que es / sea una buena persona

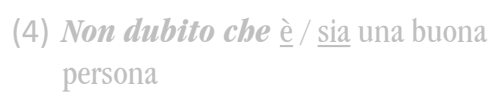

(5) Nadie duda de que el día será fantástico

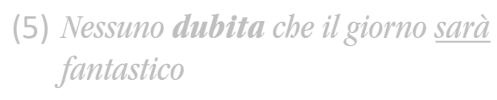

(6) Es improbable que venga a la fiesta

(6) È improbabile che venga alla festa. 
En (3) el uso del indicativo en lugar del subjuntivo enfatiza la mayor factualidad que el hablante atribuye al enunciado, mientras que en italiano el uso del modo realis en oraciones afirmativas es poco común. El caso (4), en cambio, presenta una mayor productividad en esta última lengua; en los registros informales predomina la construcción con indicativo, mientras que en niveles estilísticos superiores la negación no afecta la regencia modal del verbo. Wandruszka (2001) declara que el adverbio non "deriva una sorta di predicato quasi-affermativo che esercita un influsso immediato su modalità e modo (...) e che corrisponde ampiamente alla costruzione Sono convinto che (...)" (p. 432).

En el punto (4), el español prefiere el subjuntivo después del verbo negado cuando el hablante no quiere comprometerse con la veracidad de la información dada por una fuente externa o cuando él se plantea agregar una objeción al contenido proposicional de la frase. En el ejemplo (5), los gramáticos de ambos idiomas aceptan que el futuro sirve para resaltar la posterioridad de la oración, pues consolida la fuerza asertiva de esta: "mood choice represents possible contexts of evaluation, determined by a scale that ranges from nonrealistic, weakly realistic, realistic to totally realistic contexts" (Gielau, 2015, p. 27). Finalmente, el punto (6) exige el subjuntivo debido esencialmente a que su valor semántico corresponde a una dimensión potencial en la que el hablante se aleja de la constatación objetiva.

En segundo lugar, con los verbos españoles negar e ignorar obtenemos alternancias similares a las mencionadas en los párrafos anteriores.

(7) Niego / ignoro que havas dicho la verdad

(8) Niega / ignora que fueran / fueron deshonestos

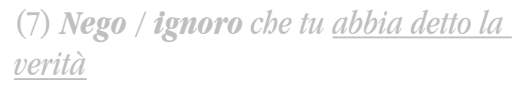
verità

(8) Nega / ignora che sono stati / siano stati disonesti

(9) Ignoramos que se aproxima /

(9) Ignoriamo che si avvicina / avvicini aproxime un meteorito. una meteorite.

(10) No niegas que conoce / conozca los (10) Non neghi che conosca i fatti? hechos?

(11) ¿No ignoras que conoce los hechos? (11) Non ignori che conosce / conosca ifatti?

El ejemplo (7) en ambas lenguas deja entrever que en aquellas construcciones en presente en las que el sujeto del enunciado y el de la 
oración coinciden "no es posible diversificar el compromiso sobre la verdad de la proposición aseverada y, en tal caso, el empleo de indicativo resulta agramatical" (Ridruejo, 1999, p. 3223). El indicativo es admitido en aquellas oraciones (8) donde el hablante se distingue del sujeto y además "egli vuole far capire che considera alla stregua di un fatto il contenuto della frase dipendente negato dal rispettivo soggetto (qualora compaia il congiuntivo resta invece indeciso se il parlante condivida o meno l'affermazione della persona denotata dal SOGGETTO della predicazione)" (Wandruszka, 2001, p. 433). Se podría sintetizar entonces que "prototípicamente en las construcciones con subjuntivo, el sujeto de la oración principal no tiene control sobre el segundo sujeto, por ende, no puede determinar si el evento sucederá/sucedió o no" (Cueva, 2018, p.78).

Al mismo tiempo, las oraciones en las que la negación léxica se anuda a la negación sintáctica (no / non) presentan una suerte de reajuste léxico en el que se añade un mayor nivel de confiabilidad a la expresión $(10,11)$. Esto provoca que verbos como negar, ignorar e ignorare conquisten el significado de afirmar, saber y sapere respectivamente. Empero, un elemento contrastante a esta lógica es el verbo negare, en el cual "la negazione non ha alcun influsso sulla scelta del modo e (...) che viene di norma associato al congiuntivo" (Wandruszka, 2001, p. 433).

En tercer lugar, existen otras proposiciones subordinadas completivas que dependen de predicados que expresan conocimiento. Se consideren los siguientes ejemplos:

(12) Cree / piensa que el niño llegó tarde.

\section{(12a) Crede / pensa che il bambino sia arrivato tardi}

\section{(12b) Crede/ pensa che il bambino è arrivato} tardi

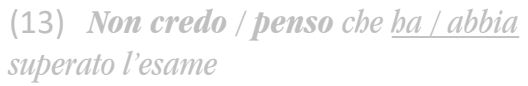
aprobado el examen

(14) ¿Crees / piensas que es / sea interesante?

(15) Creo que no es una buena persona

Para empezar, el italiano requiere el subjuntivo en la mayoría de los contextos sintácticos con los vocablos credere / creer (12a, 13, 14), pero en ciertos casos permite el uso del indicativo $(12 \mathrm{~b}, 14,15)$ cuando se quiere expresar una creencia ligeramente debilitada. Vale la pena señalar que, aunque 
este modo se usa principalmente en registros informales, no es un fenómeno sociolingüístico moderno, "già dal secondo Ottocento l'uso dell'indicativo in una proposizione completiva non è raro in scrittori che intendano accostarsi al parlato, specie quando si trovino a riprodurre le battute di un personaggio" (Serianni \& Antonelli, 2011, p. 107). Asimismo, el razonamiento adoptado en (7c) debe replicarse en (12b) y (14), ya que el indicativo sirve para señalar la creencia común entre el sujeto de la oración y el enunciador. Por su parte, el español solo permite la modalidad de irrealidad cuando existe un inductor de polaridad de la oración principal como la negación (13) o la interrogación (14). La forma creer que + no + verbo también suele requerir el indicativo.

(16) Pienso que este problema se (16) Penso che questo problema si risolverà resolverá

(17) Pensé que la vida era bella.

\section{(17b) Pensai che la vita era bella.}

Finalmente, con respecto a la elección modal después del verbo pensar / pensare, se mantienen ciertas distribuciones anteriores. Wandruszka (2001) recuerda que los verbos italianos pensare y credere están estrechamente relacionados semánticamente y que en oraciones negativas (13), interrogativas (14) e hipotéticas (17a) es preferible su uso con el subjuntivo. Una diferencia notable entre los dos verbos es la coincidencia de pensare con el significado de riflettere sul fatto che (17b) donde el indicativo es concebible. Asimismo, se admiten el futuro y el condicional para resaltar la posterioridad de la cláusula subordinada (16). Por su parte, el español rara vez admite el subjuntivo en oraciones afirmativas, ya que en ellas el hablante "indicates that he considers the proposition (...) to be true, that is, a proposition which expresses a real state of affairs" (Haverkate, 2002, p. 60), sea porque es verdadera 0 porque refleja una situación experimentada ${ }^{3}$. En la negación, dado que el contenido del enunciado ya no es fáctico y el sujeto del predicado epistémico no está en posición de evaluar el valor de verdad de la proposición subordinada, el subjuntivo es preferible.

3 Una oración que podría ejemplificar la posibilidad del subjuntivo en oraciones afirmativas es la propuesta por la RAE (2010) en la que la se expresa una posición tomada por el hablante o una decisión: He pensado que sea usted el que se encargue del asunto (Gallego, Adelaida). 
(19) Ella no sabía que tu tenías / tuvieras un carro

(20)

(Yo) No sabía que eras / fueras

el jefe

Con respecto al verbo saber / sapere, es menester mencionar que este se inserta en la categoría de los verbos fácticos, ya que comunica un hecho conocido al sujeto. Dicho esto, el indicativo es el modo no marcado en ambos idiomas. La negación ocasiona polaridad en la selección modal: en (18) el verbo adquiere el significado de enterarse de, accorgersi y por ende privilegia el uso del indicativo, mientras que en (19) el subjuntivo puede emplearse si el punto de vista del hablante difiere de la perspectiva del sujeto de la predicación. Con el indicativo, el hablante es consciente de la facticidad del contenido de la oración, mientras que con el modo irrealis se destaca el desconocimiento del hablante sobre el tema de la proposición. Según Bustos (1974), el hablante de la lengua española selecciona el subjuntivo cuando no está de acuerdo con la veracidad de la oración y explicita su disconformidad lingüísticamente.

Wandruszka (2001) puntualiza que cuando el hablante y el sujeto de la oración principal son idénticos en la primera persona del singular, es posible señalar la misma disociación entre las dos entidades siempre que el momento del evento y el del enunciado no coincidan (20): "la persona denotata dal soggetto della predicazione può cioè sapere adesso ciò che non sapeva ancora al momento descritto nel passato" (Wandruszka, 2001, p. 442). En efecto, el indicativo expresa un hecho que el hablante-sujeto ignoraba pero que ahora conoce, mientras que el subjuntivo revela una duda 0 incluso un conflicto con el interlocutor o el sujeto de la oración subordinada.

\section{Predicados volitivos (subjuntivo deóntico)}

Las proposiciones sustantivas que expresan un deseo o mandato requieren habitualmente el subjuntivo:

desiderative predicates are subject to the rule that the subordinate clause is marked by infinitive complementation if the subjects of the matrix and the subordinate clause are coreferential. When no referential identity between the subjects holds, the use of the subjunctive is obligatory. (Haverkate, 2002, p. 93) 
Como indica el autor, solo cuando existe una correspondencia entre el sujeto de la oración principal y la subordinada es posible la alternativa con infinitivo.

\begin{tabular}{|c|c:c|c:c|c|}
\hline ITALIAN0 & I & S & ESPAÑOL & I & S \\
\hline $\begin{array}{c}\text { Accettare, } \\
\text { ammettere }\end{array}$ & & $\mathrm{X}$ & Aceptar, admitir & & $\mathrm{X}$ \\
\hline Volere & $\mathrm{X}$ & $\mathrm{X}$ & Querer & $\mathrm{X}$ \\
\hline Decidere & $\mathrm{X}$ & $\mathrm{X}$ & Decidir & $\mathrm{X}$ & $\mathrm{X}$ \\
\hline Esigere, ordinare & $\mathrm{X}$ & $\mathrm{X}$ & Exigir, Ordenar & $\mathrm{X}$ \\
\hline Impedire & & $\mathrm{X}$ & Impedir & $\mathrm{X}$ \\
\hline $\begin{array}{c}\text { Lasciare, } \\
\text { permettere }\end{array}$ & & $\mathrm{X}$ & Dejar, Permitir & & $\mathrm{X}$ \\
\hline Preferire & & $\mathrm{X}$ & Preferir & & $\mathrm{X}$ \\
\hline Sperare & $\mathrm{X}$ & $\mathrm{X}$ & Esperar & $\mathrm{X}$ & $\mathrm{X}$ \\
\hline
\end{tabular}

Figura 2. Verbos volitivos, I (indicativo), S (subjuntivo)

En italiano, el subjuntivo también aparece por norma cuando el verbo matriz es uno de la lista presentada anteriormente; no obstante, la lengua hablada del centro-sur permite la alternancia con el indicativo (21). Este fenómeno se puede rastrear sobre todo en la segunda persona del singular y del plural. Otros verbos, sin embargo, pueden sufrir cambios semánticos dependiendo del modo utilizado. El verbo decidir / decidere, por ejemplo, adquiere el significado de concluir cuando es conjugado al indicativo (22).

(21) Espero que ganes / ganarás el (21) Spero che tu superi / supererai l'esame examen

(22) Decidió que era mejor estudiar (22) Ha deciso che era meglio studiare

El verbo esperar / sperare también genera contextos opacos. El indicativo es utilizado cuando se hace referencia a un proceso localizado en el futuro (21), lo que se explica por el hecho de que el modo de realidad supone una mayor aseveración o certeza sobre lo que se considera que sucederá. Esperar alude no solo a la voluntad, sino además a la previsión sobre la factibilidad del enunciado. 


\section{Proposiciones relativas}

Las subordinadas relativas se clasifican en explicativas y especificativas. $\mathrm{Su}$ principal diferencia radica en que las primeras añaden información complementaria a un sintagma nominal y suelen estar separadas por una pausa; las segundas, por otro lado, se adhieren directamente a la oración principal (antecedente) y la modifican restringiendo su extensión. La selección del modo en estos dos tipos de hipotaxis se analiza a continuación.

\section{Aposición explicativa}

En un contexto no restrictivo, la elección del indicativo es bastante simple considerando que la descripción presentada en la cláusula subordinada representa un hecho real (Pérez, 1999, p. 3257; Haverkate, 2002, p. 184; Bermejo, 2009, p. 59). Se pueden identificar dos excepciones en español: en primer lugar, el subjuntivo aparece en fórmulas con un optativo ritual en el cual el antecedente no proviene de la estructura relativa, sino de un inductor optativo implícito (23). Un segundo evento de oración explicativa en subjuntivo se puede revelar en el ejemplo (24) donde la presencia de fuera destaca una nueva información que debería conocerse para la comprensión total de la oración principal. La única irregularidad en italiano se encuentra en el modelo (23) al que se aplica la misma explicación.

(23) Tu madre, que Dios la bendiga, era una mujer muy fuerte.

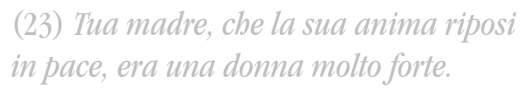

(24) Mi abuelo, quien fuera el presidente del sindicato, murió luchando por sus derechos.

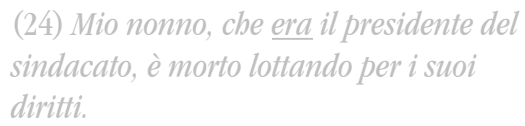

\section{Aposición especificativa}

De manera prototípica, la alternancia de subjuntivo / indicativo está directamente ligada a los conceptos de especificidad (indeterminación), referencialidad y factualidad del antecedente. El indicativo se usa cuando "el SN es especifico y, por lo tanto, cuando la entidad que designa existe en un mundo concreto del universo del discurso (25); por el contrario, se construye con subjuntivo cuando el SN es inespecífico y la entidad no existe (26), o el emisor no puede o no quiere afirmar la existencia" (Pérez, 1999, p. 3256). Dentro de los sintagmas nominales que introducen el indicativo se evidencian los sustantivos con artículos definidos o con adjetivos demostrativos (27). El antecedente indefinido puede desencadenar tanto la aparición del subjuntivo como del indicativo y suele estar compuesto por un artículo indefinido (25, 
26), un pronombre o un adjetivo indefinido (algo / qualcosa, alguien / qualcuno...).

(25) Busco una película que me gusta.

(25) Cerco un film che mi piace

(26) Busco una película que me guste.

(26) Cerco un film che mi piaccia

(27) Busco la / esa película que me gusta.

(27) Cerco il / quel film che mi piace

La alternancia modal solo es posible si se puede dar fe de la aparición de un elemento que cree contextos opacos, esto es, de un modalizador que interrumpa la correferencialidad del SN y que genere ambigüedad. Se exponen a continuación algunos nexos que permiten la manifestación del modo irrealis:

Primero, el subjuntivo en oraciones relativas puede ser introducido por predicados con valor volitivo (28) como querer / volere, desear / desiderare, decidir / decidere, necesitar / avere bisogno di, e buscar / cercare. En segundo lugar, los verbos modales poder / potere, deber, tener que, también son inductores de opacidad cuando "estos elementos asumen un valor deóntico (de obligación, necesidad o permiso); (29) pero también cuando tienen un valor epistémico (de probabilidad o eventualidad); (30) 0 un valor dinámico (de capacidad) (31)" (Pérez, 1999, p. 3261). En las opciones (30, 31 , aunque las oraciones con el subjuntivo son gramaticalmente correctas, el indicativo es preferido por los hablantes italianos para la determinación de la oración. La forma (29a) no es posible a menos que use un indefinido como qualsiasi (29b).

(28) Queremos comprar un libro que trate / trata sobre el subjuntivo.

(29) Puedes escoger el libro que quieras / quieres

(30) Puede que yo tenga una evidencia que te inculpe / inculpa

(31) Diego puede leer libros que estén / están escritos en sánscrito

\section{(28) Vogliamo comprare un libro che tratti / tratta del congiuntivo.}

(29b) Puoi scegliere qualsiasi libro tu $\underline{\text { voglia }}$

(30) Può darsi che io abbia una prova che ti incrimini / incrimina 
En tercer lugar, es menester mencionar que los indefinidos negativos hacen las veces de modalizadores: las formas nadie, nada, ningún, ninguno, ninguna, acompañan a los antecedentes inespecíficos que aluden a una clase vacía haciendo que el subjuntivo se vuelva obligatorio. Una interpretación similar se puede encontrar en italiano, la referencialidad o verdad de la oración relativa y de su antecedente puede ser cuestionada a través de la negación: nessuno, niente, nulla. En estos casos en los que se denota un conjunto vacío, el modo predeterminado es el subjuntivo (32a), pero en algunos contextos no controlados también se usa su contraparte (32b).

En cuarto lugar, ambas lenguas solo aceptan el subjuntivo cuando los inductores son cuantificadores indefinidos como cualquiera / qualunque - qualsiasi, quienquiera / chiunque, dondequiera / dovunque (33). Su presencia se explica por el hecho de que estas palabras anuncian un conjunto, una globalidad en la que los detalles pasan a un segundo plano y en la que se expresa una idea potencialmente indefinida. Algo similar ocurre con los adjetivos en grado superlativo (34a) donde no se alude a una entidad definida, sino a una generalidad (la categoría uomini / hombres) y donde se vislumbra la falta de certeza y compromiso del hablante con su enunciado. Desde una perspectiva pragmática, Haverkate (2002) señala que con esta construcción

it may be difficult for the speaker to decide which entity is the optimum representative of the field. The speaker may be in doubt, for instance, as to attributing a superlative value to the entity selected or to ascertaining whether or not the entity is the first one of a set or series. (p. 191)

Con el indicativo, en cambio, el hablante implica que ya ha analizado todas las posibilidades y que está seguro de que su percepción es acorde con el mundo real. El uso de este modo en italiano no posee una connotación específica, sino que refleja variación de carácter diafásico y diastrático (34b).

(32) No encuentro ninguno que valga la pena

(32a) Non trovo nessuno che valga la pena

(32b) Non trovo nessuno che vale la pena

(33) Dondequiera que vavas, te (33) Dovunque tu vada, ti troverò encontraré

(34a) Pedro es el hombre más rico que (34a) Pedro è l'uomo più ricco che conosca conozca

(34b) Pedro es el hombre más rico que (34b) Pedro è l'uomo più ricco che conosco $\underline{\text { conozco }}$

Finalmente, dentro de los creadores de contextos opacos en frases relativas se destacan las frases interrogativas y las condicionales 
introducidas por el complementador se/si y quando/cuando. En ambos casos el valor dubitativo de la frase permite múltiples lecturas y por lo tanto son fuente de polaridad modal. En consecuencia, las formas (35) y (37) son igualmente correctas si se tienen en cuenta los criterios de existencia, especificidad, realidad o potencialidad. Sin embargo, el indicativo es esencial cuando el individuo es definido o especificado por uno de los medios indicados al comienzo de este apartado (artículos demostrativos definitivos; 36, 38).

(35) ¿Han visto un libro que tenga / tiene un prólogo de Gabriel García Márquez?

(36) ¿Quién es la mujer que te está / esté* esperando?

(37) Si escribes un texto que exponga / expone los usos del subjuntivo, idímelo!

(38) Llámame si llega el paquete que contiene / contenga* los libros
(35) Avete visto un libro che abbia / ha un prologo di Gabriel García Márquez?

(36) Chi è la donna che ti sta / stia* aspettando?

(37) Se scrivi un testo che presenta Lpresenti gli usi del congiuntivo, dimmelo!

\section{Proposiciones adverbiales}

El concepto de subordinación adverbial representa un problema terminológico para los estudios gramaticales contemporáneos. Con propósitos metodológicos y en contraste con el concepto de subordinación relativa y completiva, se utilizará el término subordinación adverbial para referirse a construcciones temporales, concesivas, causales, finales y comparativas. Se omitirán aquellas proposiciones de difícil clasificación (cláusulas modales y consecutivas) 0 aquellas que no pertenezcan al grupo de subordinación, sino al grupo de coordinación (cláusulas condicionales).

\section{Cláusulas subordinadas temporales}

La Real Academia Española (2010) afirma que las partículas temporales cuando (adverbio relativo) (39), mientras (conjunción con el sentido de durante el tiempo que) (40), y después de que (41) admiten tanto el subjuntivo como el indicativo $0^{4}$ : "el subjuntivo requiere un inductor modal de valor prospectivo, como el imperativo, el futuro" (p. 488) o el presente con valor de futuro.

3 Sin embargo, no admiten el futuro o el condicional. 
(39a) Cuando vengas, bebemos algo.

(39b) Cuando hayas estudiado, te daré un helado.

(39c) Ven a mi casa cuando salga el sol

(39d) Siempre vienes a mi casa cuando sale el sol.

(39e) Cuando Diego quisiera decirme la verdad, solo tendría que llamarme

(40a) Mientras vivas conmigo, no te faltará nada

(40b) Ahorro dinero mientras vivo con mis padres

(41a) Después de que comas, lo hablaremos.

(41b) El accidente ocurrió después de que vino / viniera a mi casa. (39a) Quando verrai, berremo qualcosa.

(39b) Quando avrai studiato, ti darò un gelato.

\section{(39c) Vieni (imperativo) a casa mia quando sorge il sole}

(39d) Vieni (presente) sempre a casa mia quando sorge il sole

(39e) Quando Diego volesse dirmi la verità, solo dovrebbe chiamarmi

(40a) Mentre vivrai con me, non ti mancherà nulla

(40b) Risparmio dei soldi mentre vivo con i miei genitori

(41a) Dopo che avrai mangiato, ne parleremo.

(41b) L'incidente è successo dopo che è venuto a casa mia

Las traducciones italianas no permiten el modo de irrealidad en estas condiciones, excepto en el modelo (39e) en el que "si vuole esprimere l'ipoteticità (possibilità 0 impossibilità) della temporale" (Giusti, 2001, p. 721). En los ejemplos enumerados anteriormente se observa que cuando el subjuntivo tiene un valor prospectivo, el italiano prefiere el futuro anterior o simple. Además, construcciones como las de (39d) y (40b), que carecen de valor anticipatorio, comparten el indicativo en ambos idiomas. La expresión conjuntiva después de que resulta más polivalente con respecto a la elección del modo en español. De hecho, al describir hechos presentes o pasados, ambos paradigmas modales son posibles sin ningún cambio de tipo semántico (41a, 41b). Cabe mencionar que las frases en español "si costruiscono con l'indicativo se designano avvenimenti fattuali, cioè passati, presenti 0 abituali (42b, 43b), e con il congiuntivo se rinviano a situazioni posteriori non fattuali, cioè eventuali 0 virtuali (42a, 43a)" (Bermejo, 2008, p. 63). Este es el caso de en cuanto, hasta que y antes de que. Los enlaces a medida que y después de que admiten ambos modos bajo el mismo principio. Mientras tanto, el italiano excluye este tipo de variación modal con los últimos conectivos (42a, 42b, 43). 
(42a) A medida que entren les entregas las revistas

(42b) A medida que entraron les entregaron las revistas.

(43a) Nos vemos después de que hable con él (42a) Man mano che entrano, gli consegni le riviste

(42b) Man mano che sono entrati, gli hanno consegnato le riviste

(43) Ci vediamo dopo che parlo / avrò parlato con lui

(43b) Nos vimos después de que hablé con él

\section{Clausulas subordinadas concesivas}

Algunos conectores subordinados pueden optar por el indicativo ( $a$ sabiendas de que, si bien), el subjuntivo (así, por muy ... que, a riesgo de que; a pesar, aunque, para qué ...) o las dos opciones. En la última posibilidad, se recuerdan vocablos como aunque, pese a, a pesar de para el español; y anche se para el italiano. Se mencionan algunos ejemplos:

(44a) Aunque / a pesar de que

estábamos enfermos, fuimos a la fiesta

(44b) Aunque / Pese a que

estuviéramos enfermos, fuimos a la fiesta

(44c) Aunque sea / es difícil, puedes superar este problema.

(44d) No podría vencerme, aunque tuviera la fuerza de tres hombres

\begin{abstract}
(44a) Anche se eravamo malati, siamo andati alla festa. Nonostante fossimo malati.
\end{abstract}

(44b) Anche se fossimo* /eravamo malati, siamo andati alla festa

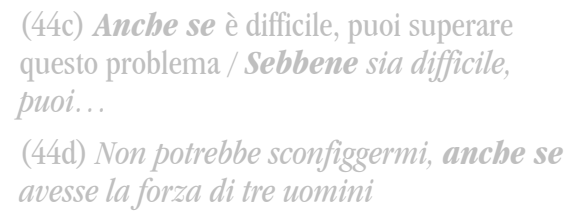

La diferencia entre la oración (44a) y (44b) radica en el grado de importancia 0 valor que se otorga al contenido de la proposición concesiva. Como bien afirman Craverns y Lunn (1991), con el indicativo se presenta la proposición como relevante, real, verdadera, nueva o inesperada, mientras que con el subjuntivo se refuerza el hecho de que si bien la información es fáctica no es importante o que, aunque puede llegar a ser factual, aún no se ha realizado. Haverkate (2002) sostiene una explicación pragmática de esta dualidad, según la cual con el subjuntivo "the propositions expressed may be true, but the speaker is not in a position to verify whether the events referred to took place in actual fact" (p. 169). El modelo (44c) podría ayudar a explicar esta posición: al usar el indicativo, el hablante legitima la complejidad del problema o se demuestra empático hacia el interlocutor, pero cuando usa el subjuntivo este se aleja de la dificultad o sugiere que no conoce muy bien los hechos. Contrariamente, en italiano la selección del subjuntivo o indicativo en las proposiciones concesivas 
no proviene del valor pragmático de la cláusula, sino de la regencia del operador sintáctico. Prandi (1996) afirma que "nella sua qualità di perno della codifica, la congiunzione subordinativa di regola seleziona il modo della forma verbale subordinata. Dopo che e anche se, ad esempio, reggono l'indicativo, mentre prima che e benché (sebbene, nonostante) reggono il congiuntivo" (p. 40). El único caso de variación modal en oraciones concesivas compartido tanto por el italiano como por el español se destaca en el ejemplo (44d), donde se inserta una proposición hipotética que describe una cuestión contrafactual.

\section{Proposiciones subordinadas causales}

Dentro de la categoría de cláusulas que establecen una relación causal con la oración principal se reconocen porque, como, ya que, puesto que, dado que, pues, visto que, en vista de que, debido a que, en lengua castellana, y siccome, visto che, in quanto, dato che, poiché, giacché, per la ragione che, en italiano. En cuanto a la forma en que se lleva a cabo este tipo de construcciones, Pérez (1999) afirma que "en términos generales, las oraciones causales exigen el modo indicativo independientemente del nexo que las introduzca y del valor concreto que asuman" (p. 3287). Esta afirmación puede extenderse a ambos idiomas.

(45a) No te dio el dulce porque estabas triste

(45) Non ti ba dato la caramella perché eri triste

(45b) No te dio el dulce porque estuvieras triste

(46) Te dio el dulce no porque estuvieras triste, sino porque te ama.

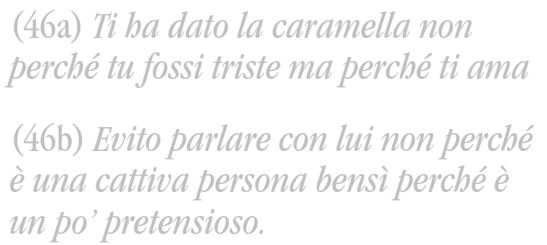

Sin embargo, cabe destacar que las conjunciones porque y perché permiten el subjuntivo cuando se encuentran dentro de una oración negativa. En el caso de porque, el uso del modo irrealis indica la negación del contenido introducido por la hipotaxis, mientras que con el indicativo es el verbo principal el que resulta negado. En consecuencia, en el ejemplo (45a) se dice que la causa de no darle el dulce fue la tristeza del interlocutor, mientras que en (45b) se especifica que la razón por la que no le dio el caramelo al individuo no fue porque se sintiera decaído, sino por una razón no especificada. En cuanto a perché, el uso del subjuntivo no es posible, ya que la estructura non + verbo principale + perché exige el indicativo (45). 
Por su parte, cuando el adverbio negativo precede inmediatamente al porque, el subjuntivo es obligatorio (46), ya que la causa se considera ficticia: "La non effettività o la non centralità della causa, oppure la negazione completa di essa, appare manifesta se subito dopo, mediante sino, il parlante esprime la causa che ritiene veramente effettiva" (Bermejo, 2008, p. 119). La lengua dantesca, aunque admite esta misma interpretación (46a), acepta además el indicativo en un registro coloquial cuando el hecho negado es considerado verdadero (46b).

\section{Proposiciones subordinadas finales}

El grupo de conectivos que manifiesta el propósito o intención de la oración principal constituye un conjunto homogéneo y bien definido con respecto a la elección modal. Bertuccelli (2001) afirma que "la proposizione finale sottintende un atteggiamento di tipo volitivo-intenzionale sulla realizzazione del quale non può esserci certezza fattuale" (p. 818). Por tanto, el modo en las oraciones explícitas es siempre el subjuntivo en las dos lenguas estudiadas. Entre los nexos más habituales en las finales españolas se encuentran para que, que, a fin de que, con el fin de que, con el objetivo de que, con el propósito de que. En italiano, por otro lado, se pueden mencionar conjunciones como perché, affinché, che, e acciocché.

\section{Proposiciones comparativas}

Normalmente, la oración comparativa se define como una proposición que compara el contenido del subordinado y la oración principal en términos de superioridad, inferioridad o igualdad. Para confirmar que la cláusula pertenece a esta tipología, se debe hacer una comparación tanto del componente sintáctico como semántico.

(47) Sara critica más de lo que hace

(48a) Trabaja tanto como puedas

(48b) Trabajas tanto como puedes

(48c) Trabajaré tanto como pueda

(48d) Cuanto más crece, más bello se vuelve

(48e) Cuanto más crezcas, más entenderás

\section{(47a) Sara critica più di quanto fa}

\section{(47b) Sara critica più di quanto \\ faccia}

(48a) Lavora più che puoi

(48b) Pietro è (tanto) amabile quanto simpatico

(48c) Lavorerò il più possibile

(48d) Più cresce, più bello diventa

(48e) Più cresci, più capirai 
En cuanto a la preferencia modal de este tipo de enunciados, el ejemplo (47) exhibe una disimetría entre su funcionamiento en español y en italiano. En el primer idioma, el nexo comparativo de desigualdad más que introduce el indicativo siempre que la comparación se haga entre dos acciones verbales. El italiano, en cambio, muestra una polivalencia modal que parte solo de la preferencia estilística del hablante, es decir, de la formalidad (47b) 0 informalidad (47a) de la conversación: "L'uso dell'indicativo è marca di espressione poco accurata per Belletti (2001 II, p. 838): 'stile dimesso' e per Serianni (1988, p. 519): 'oggi caratteristico del livello meno formale; ma un tempo l'indicativo era adoperato senza restrizioni di sorta'” (Bermejo, 2008, p. 214). Sin embargo, la construcción più / meno di quello che impone siempre el indicativo (49c).

Por lo demás, el resto de las construcciones comparativas de desigualdad en español permiten la alternancia modal. Como ocurre con las oraciones relativas, la mayor discrepancia semántica radica en la especificidad del contenido. Mientras que con el indicativo la cláusula adquiere un valor fáctico y específico en el que el hablante se compromete con el enunciado (48d), el subjuntivo supone una proposición no específica, hipotética o virtual (48e). Las comparaciones de igualdad optan por el indicativo (48b), pero no raramente admiten el modo irrealis: este es el caso de las construcciones introducidas por un verbo en futuro (48c) o en imperativo (48a). Finalmente, en italiano, según Belletti (2001) "la comparativa di uguaglianza è al modo indicativo, e la comparativa di disuguaglianza è al modo congiuntivo (48b). Lindicativo è possibile marginalmente nella comparativa di disuguaglianza, ma il congiuntivo è sistematicamente escluso da quella di uguaglianza (48b)" (p. 844).

\section{Conclusiones}

Una caracterización del subjuntivo basada puramente en el componente sintáctico no es suficiente para explicar las múltiples realizaciones de este modo verbal. Existen contextos opacos en los que el subjuntivo puede tener una variante con el indicativo o incluso con el condicional: diferentes tipos de modalizadores como la negación, la interrogación, los indefinidos (artículos, adjetivos, pronombres), las conjunciones pueden introducir el subjuntivo. Los dos primeros inductores (negación e interrogación) tienen efecto en todas las proposiciones; el subjuntivo inducido por los indefinidos es operativo sobre todo en las oraciones relativas; las conjunciones, por otro lado, tienen un mayor campo de acción dentro de las proposiciones adverbiales. En otros casos, como en oraciones complementarias, la actitud, el nivel de implicación o certeza y la valoración de la verdad realizada por el hablante pueden determinar la selección modal. 
Es además claro que, aunque la yuxtaposición del subjuntivo con la connotación de irrealidad y del indicativo con el de realidad no es del todo exacta, la mayoría de los casos en los que aparece el primer modo están impregnados por un sentido de potencialidad, indeterminación, inexistencia, falta de afirmación o duda. Es menester agregar que, mientras en español la polaridad subjuntivo / indicativo encuentra generalmente un valor expresivo (pragmática), en italiano el uso del modo irrealis en contextos que demandan su contraparte se interpreta como una variante sociolingüística diastrática y diafásicamente marcada.

Por último, hay que decir que este estudio, dada su envergadura, ha dejado fuera muchos aspectos que es necesario contrastar en el futuro. Algunos de los puntos críticos para investigaciones posteriores es la caracterización de los usos de interrogativos indirectos, las diferencias y similitudes entre los dos lenguajes en cuanto al subjuntivo fáctico, entre otros.

\section{Referencias}

Battaglia, S.\& Pernicone, V. (1980). La grammatica italiana. Loescher.

Belletti A. (2001). Funzioni delle frasi subordinate: Comparative, In A. Cardinaletti, L. Renzi, \& G. Salvi, Grande Grammatica Italiana di Consultazione (pp. 832853). Il Mulino.

Bello, A. (1857). Gramática de la lengua castellana destinada al uso de los americanos. (4.a ed.). Echeverry Hermanos.

Bermejo, F. (2008). Le subordinate avverbiali: Uno studio contrastivo spagnoloitaliano. Bononia University Press.

Bermejo, F. (2009). Le relative spagnole e italiane. Celid.

Berruto, G. (1987), Sociolinguistica dell'italiano contemporaneo. Carocci.

Berruto, G. (2010). Italiano standard. Enciclopedia dell' italiano Treccani.

Bertucelli, M. (2001) Funzioni delle frasi subordinate: Finali, , In A. Cardinaletti, L. R enzi, \& G. Salvi, Grande Grammatica Italiana di Consultazione (pp. 818825). Il Mulino.

Bustos, A. (1974) A syntactic correlate of semantic and pragmatic relations: The subjunctive mood in Spanish. University of Illinois.

Bustos, E. (1986). Pragmática del español: negación, cuantificación y modo. Universidad Nacional de Educación a Larga Distancia. 
Cravens, T., \& Lunn, P. (1991). A contextual reconsideration of the Spanish -ra "indicative". In S. Fleischman, \& L. Waugh, Discourse Pragmatics and The Verb. Routledge.

Cueva, A. (2018). Aproximación al modo subjuntivo del español desde una perspectiva multivariable. Folios, (48), 73-84.

Dardano, M., Trifone, P. (1988). La lingua italiana. Zanichelli.

Falcinelli, A. (2015). Modalidad epistémica y adverbios de duda: el caso de magari en italiano. Linguae, 14(2), 29-46.

Fogarasi, M. (1983). Grammatica italiana del Novecento. Bulzoni.

Fornaciari, R. (1879). Grammatica della lingua italiana. Sansoni.

Giannakidou, A. (2009). The Dependency of the Subjunctive Revisited: Temporal Semantics and Polarity. Lingua, 119, 1883-1908. http://home.uchicago. edu/ giannaki/pubs/LinguaMood2009.final.pdf

Gielau, A. (2015). Mood Distribution and the CP Domain ff Subjunctive Clauses in Spanish. (PhD thesis), University of Iowa. https://doi.org/10.17077/etd. bpl4g56e

Gili, G. (1980). Curso superior de sintaxis española. Bibliograf.

Giorgi, A. (2009). Toward A Syntax of the Subjunctive Mood. Lingua, 119(12), 18371858.

Giusti, G. (2001). Frasi avverbiali: temporali, causali e consecutive. In A. Cardinaletti, L. Renzi, \& S. Giampaolo, Grande Grammatica Italiana di Consultazione (pp. 720-784). Il Mulino.

Haverkate, H. (2002). The Syntax, Semantics and Pragmatics of Spanish Mood (Pragmatics \& Beyond New Series). John Benjamins Publishing.

Lenz, R. (1920). La oración y sus partes. Centro de Estudios Históricos, Junta para la Ampliación de Estudios e Investigaciones Científicas, RFE.

Pérez, M. (1999). El modo en las subordinadas relativas y adverbiales. En I. Bosque \& V. Demonte (dirs.), Gramática descriptiva de la lengua española. Vol. 2 (pp. 3253-3322). Espasa Calpe.

Prandi, M. (1996). La subordinazione non completiva. Un frammento di grammatica filosofica. Studi Italiani di Linguistica Teorica e Applicata, 25, 1.

Real Academia Española -RAE-. (2009). Nueva gramática de la lengua española. ESPASA. 
Real Academia Española -RAE-. (2010). Manual de la nueva gramática de la lengua española. ESPASA.

Ridruejo, E. (1999). Modo y modalidad. El modo en las subordinadas sustantivas. En I. Bosque \& V. Demonte (dirs.), Gramática descriptiva de la lengua española. Vol. 2 (pp. 3209-3251). Espasa Calpe.

Rojo, G. (1976). La correlación temporal. Verba, (3), 65-89.

Sabatini, F. (1985). L'italiano dell'uso medio: una realtà tra le varietà linguistiche italiane, En: Geschichte und Gegenwart, hrsg. von Günter Holtus, Edgar Radtke (dirs.), Gesprochenes Italienisch. pp. 154-185. Tübingen, Narr.

Salvá, V. (1835). Gramática de la lengua castellana según ahora se habla. Librería de los SS.

Sensini, M. (1990). La grammatica della lingua italiana. Mondadori.

Serianni, L. \& Antonelli, G. (2011). Manuale di linguistica italiana: storia, attualità, grammatica. Pearson.

Sorrento, L. (1951). Sintassi romanza. Istituto Editoriale Cisalpino

Stewart D. (2002): Il congiuntivo italiano: modo della realtà? Uno sguardo al congiuntivo nelle grammatiche italiane moderne, in L. Schena, M. Prandi, M. Mazzoleni (a cura di), Intorno al congiuntivo: storia, tipologia, traduzione, Bologna: CLUEB, p. 105-122. - ISBN: 8849118821.

Vanelli, L. (2001). La concordanza dei tempi. In A. Cardinaletti, L. Renzi, \& S. Giampaolo, Grande Grammatica Italiana di Consultazione (pp. 611632). Il Mulino.

Vásquez, J. (2013). Los modos verbales del español actual. Lingüística y Literatura, $255-271$.

Wandruszka, U. (2001). Frasi subordinate al congiuntivo. In A. Cardinaletti, L. Renzi, \& G. Salvi, Grande Grammatica Italiana di Consultazione (pp. 415-481). Il Mulino.

Zambaldi, F. (1905). Grammatica della lingua italiana. Sonzogno. 